\title{
Soft Skills wanted?! - (Arbeits)Fähigkeiten und Fähigkeitsanforderungen am Arbeitsplatz beschreiben mit dem Fähigkeitsrating Mini-ICF-APP
}

\author{
Beate Muschalla ${ }^{1}$ \\ Angenommen: 26. März 2021 / Online publiziert: 21. April 2021 \\ (c) Der/die Autor(en) 2021
}

\section{Zusammenfassung}

Dieser Beitrag der Zeitschrift Gruppe. Interaktion. Organisation. (GIO) informiert über ein etabliertes Standardkonzept zur fähigkeitsorientierten Arbeitsanforderungs- und Arbeitsfähigkeitsbeschreibung, welches sich im Rahmen der Arbeitsfähigkeitsbegutachtung bewährt hat. Es beschreibt die Fremd- und Selbstbeschreibung psychischer Fähigkeiten sowie die psychischen Arbeitsanforderungen.

Arbeitsplatz- und Aufgabenzuweisungen und auch betriebliche Wiedereingliederungen von Mitarbeitenden mit (chronischen) Gesundheitsbeeinträchtigungen erfordern Kenntnisse über die (Arbeits-)Fähigkeiten von Mitarbeitenden. Bei psychischen Arbeitsanforderungen ist es notwendig, das psychische Fähigkeitsprofil Mitarbeitender zu kennen und mit den Anforderungen abzugleichen.

Das Mini-ICF Rating für Aktivitäts- und Partizipationsstörungen bei psychischen Störungen (Mini-ICF-APP) ist ein international validiertes und in der Arbeitsfähigkeitsbeschreibung und Sozialmedizin etabliertes Assessment zur Beschreibung von Fähigkeitsniveaus und -profilen auf 13 psychischen Fähigkeitsdimensionen. Es wird in Leitlinien (AWMF, DRV, SGPP) zur Verwendung empfohlen. Die 13 Fähigkeitsdimensionen ermöglichen einen Abgleich des Fähigkeitsprofils der Person mit dem Anforderungsprofil des Arbeitsplatzes. Die zu beurteilenden Fähigkeitsdimensionen sind (1) Fähigkeit zur Anpassung an Regeln und Routinen, (2) Fähigkeit zur Planung und Strukturierung von Aufgaben, (3) Flexibilität und Umstellungsfähigkeit, (4) Kompetenz- und Wissensanwendung, (5) Entscheidungs- und Urteilsfähigkeit, (6) Proaktivität und Spontanaktivitäten, (7) Widerstands- und Durchhaltefähigkeit, (8) Selbstbehauptungsfähigkeit, (9) Konversation und Kontaktfähigkeit zu Dritten, (10) Gruppenfähigkeit, (11) Fähigkeit zu engen dyadischen Beziehungen, (12) Fähigkeit zur Selbstpflege und Selbstversorgung, und (13) Mobilität und Verkehrsfähigkeit.

Neben dem Fähigkeiten-Fremdrating sind weitere Instrumentenversionen auf Basis dieses Fähigkeitskonzepts entstanden: eine Fähigkeiten-Selbstratingversion (Mini-ICF-APP-S), und eine Version zur fähigkeitsorientierten Arbeitsplatzbeschreibung (Mini-ICF-APP-W) für die Herstellung passender Arbeitsplätze im Sinne des Arbeitsschutzgesetzes ( 5 ArbSchG) (Muschalla 2020).

Schlüsselwörter Fähigkeiten · Soft Skills · Arbeitsfähigkeit · Psychische Gesundheit · Psychische Erkrankungen · ICF · Person-Job-Fit

Dieser Beitrag basiert in Teilen auf einer vorangegangenen Publikation (Muschalla 2019). Er ist im Hinblick auf die Zielgruppe der Arbeits-, Organisations- und Personalpsychologie-Interessierten weiterentwickelt und modifiziert und mit neuen Aspekten ergänzt worden. In Beispielen wurden abwechselnd männliche und weibliche Formen genutzt. Es sind grundsätzlich alle Geschlechter gleichermaßen gemeint.
Prof. Dr. Beate Muschalla

b.muschalla@tu-braunschweig.de

1 Institut für Psychologie, Abteilung Klinische Psychologie, Psychotherapie und Diagnostik, Technische Universität Braunschweig, Humboldtstraße 33, 38106 Braunschweig, Deutschland 


\title{
Soft skills wanted?! - Describing work ability and work demands with the capacity concept of the Mini-ICF-APP
}

\begin{abstract}
This article in Gruppe. Interaktion. Organisation. (GIO) introduces the rating for capacity disorders and capacity demands at work, the Mini-ICF-APP. An observer- and a self-rating for employees' capacities, and a rating for capacity demands of the workplace are available.

Workplace and work demands as well as vocational reintegration of employees with (chronic) health impairments require knowledge of the (work) capacities of employees.

In view of the increasingly important psychological work demands, it is important to know the psychological capacity profile of an employee and to compare it with the work demands. This means

1. identify the strengths and, where appropriate, the limitations of the employee's capacities (e.g. problems with group integration, flexibility and decision-making capacity), and

2. describe what a workplace fitting to the person's capacities might look like (e.g. individual work, making individual customer calls, bookkeeping)

The Mini-ICF Rating for Psychological Capacity Impairments (Mini-ICF-APP, Linden et al. 2009) is an internationally validated assessment established in social medicine and work ability description to describe psychological capacity profiles by 13 psychological capacity dimensions. The 13 capacity dimensions enable a comparison of the capacity profile of the person and the demand profile of the workplace. The capacity dimensions to be assessed are (1) capacity to adapt to rules and routines, (2) capacity to plan and structure tasks, (3) flexibility, (4) application of competence and knowledge, (5) decision-making and judgement, (6) proactivity and spontaneous activities, (7) Resilience and endurance, (8) self-assertion, (9) conversation and contact with third parties, (10) group integration, (11) capacity to form close dyadic relationships, (12) self-care, and (13) mobility.

In addition to the observer rating of capacities, further versions of the instrument have been developed: a self-rating version, and a version for the capacity-oriented work demand description for the creation of suitable jobs in the sense of the German Occupational Safety and Health Act (§ 5 ArbSchG).
\end{abstract}

Keywords Capacities $\cdot$ Soft skills $\cdot$ Work ability $\cdot$ Mental health $\cdot$ Mental disorders $\cdot$ ICF $\cdot$ Person-job-fit

\section{Bedeutung psychischer Fähigkeiten in der modernen Arbeitswelt}

Im Zuge des Wandels der Arbeitswelt - von der überwiegenden Handarbeit vorindustrieller Jahrzehnte, der Kopfarbeit ab Beginn der Technisierung, hin zur heutigen computergeprägten Wissens- und Dienstleistungsgesellschaft werden immer stärker und differenzierter psychische Fähigkeiten von Mitarbeitenden verlangt (BMAS 2017; BMJV 2020).

Spezifische soziale und personale „Fähigkeiten“ - oder auch „Soft Skills“ genannt (Newman 2006; Touloumakos 2020) - werden in diesem ICF-basierten Fähigkeitskonzept verstanden als Gruppierungen von zusammen passenden Verhaltensweisen und Aktivitäten (Linden et al. 2015): Beispielsweise gehören Aktivitäten wie lächeln, jemanden ansehen, ansprechen, unverbindliche Fragen stellen und auf Fragen antworten zur Fähigkeit „Kontaktfähigkeit“. Vielfältige psychische Fähigkeiten werden tagtäglich in der Arbeit gebraucht, bspw. sich an Regeln und Routinen halten, Arbeitsabläufe strukturieren, Flexibilität, Gruppenfähigkeit oder auch Mobilität.
Personalverantwortliche, Führungskräfte und Betriebsärzt*innen müssen sich in vielfältiger Weise mit der fähigkeitsgerechten Anforderungsbeschreibung und dem Finden der Mitarbeiter*in-Anforderungspassung auseinandersetzen, bspw. bei

- Der betrieblichen Wiedereingliederung (Sozialgesetzbuch SGB IX § 167) von mehr als sechs Wochen arbeitsunfähigen oder chronisch kranken Mitarbeitenden

- Formulierung von Stellenausschreibungen und Gestaltung von Bewerbungs- und Einstellungsverfahren

- Auswahl und Zuweisung von Mitarbeitenden zu Personalentwicklungs- oder Fortbildungsmaßnahmen,

- psychischen Gefährdungsbeurteilung ( $\$ 5$ ArbSchG) und damit einer fähigkeitsorientierten Beschreibung von Arbeitsanforderungen

Für diese Zwecke müssen psychische Fähigkeiten praxistauglich beschreibbar gemacht und operationalisiert werden. Mit dem Rating für psychische Fähigkeitsbeeinträchtigungen Mini-ICF-APP (Linden et al. 2015) ist ein Konzept zur handhabbaren Beschreibung von 13 psychischen Fähigkeiten entstanden. Im Wesentlichen bildet das MiniICF-APP die heutzutage an den meisten Tätigkeitsfeldern 
erforderlichen „Soft Skills“ ab, d.h. verschiedene psychische Fähigkeiten die für Aufgabenmanagement, Interaktion und Selbststeuerung in der heutigen Lebenswelt wesentlich sind. Das Mini-ICF-APP basiert auf dem relationalen Modell der Internationalen Klassifikation der Funktionsfähigkeit, Behinderung und Gesundheit (ICF, WHO 2001) und dem Person-Job-Fit (French 1973; Edwards und van Harrison 1993).

Das Person-Job-Fit-Modell besagt, dass Arbeitszufriedenheit und -Gesundheit sich dann einstellen, wenn die Arbeitsanforderungen in Qualität und Quantität zu den Fähigkeiten der Mitarbeitenden passen (,Fit“= Passung).

Das ICF-Modell (WHO 2001) besagt, dass körperliche (Fehl)Funktionen, Fähigkeiten und Kontextfaktoren miteinander in Wechselbeziehungen stehen. Es bedeutet, dass nicht nur eine körperliche Fehlfunktion die Schwere einer Beeinträchtigung bestimmt, sondern auch die Tatsache, ob die Beeinträchtigung in einem bestimmten Kontext relevant ist. Eine Person mit Strukturierungsfähigkeit und Durchhaltefähigkeit, jedoch einer krankheitsbedingt reduzierten Grundstimmung und damit einhergehend schlechter Kontaktfähigkeit, hat möglicherweise bei einer Tätigkeit in der Buchhaltung keinerlei Beeinträchtigung. Sie kann jedoch im Verkauf gänzlich arbeitsunfähig sein. Mit dem PersonJob-Fit-Modell ausgedrückt: Die Passung von Anforderung und Fähigkeitsniveau ist in der ersten Konstellation (Regeltreue und Strukturiertheit) gegeben, in der zweiten (Kontaktfähigkeit) hingegen nicht.

Dieses Beispiel zeigt, dass Fähigkeiten(beeinträchtigungen) - im Unterschied bspw. zu einem Krankheitssymptom - immer nur in Bezug zu einem konkreten Kontext quantifizierbar sind: jemand ist nicht ,un/fähig“" per se, sondern immer nur ,un/fähig zu etwas“. Außerdem erlauben die Fähigkeitsdimensionen auch eine Ressourcenbeschreibung, d.h. über welche Fähigkeiten jemand in besonderem Maße verfügt. Analog dazu kann definiert werden, ob an einem Arbeitsplatz eine bestimmte Fähigkeit hilfreich, notwendig, oder unverzichtbar ist.

In den folgenden Kapiteln wird detaillierter auf die Anwendungsperspektive des relationalen Modelles (ICF. Person-Job-Fit) bei der Arbeitsfähigkeitsbestimmung eingegangen. Im Anschluss werden die Operationalisierungen der Fähigkeitsratings vorgestellt (Fremdrating Mini-ICFAPP, Selbstrating Mini-ICF-APP-S und Arbeitsanforderungsrating Mini-ICF-APP-W).

\section{Arbeitsfähigkeit: Eine Frage des Anforderungs-Fähigkeits-Fits!}

Hintergrund und theoretische Basis für die Arbeitsfähigkeit ist eine relationale Betrachtung von Fähigkeiten, wie man sie etwa im Person-Job Fit-Modell (Caplan et al. 1975; Ed- wards und van Harrison 1993; French 1973) findet, oder auch im modernen Gesundheitsverständnis der ICF (WHO 2001). Im Kern bedeuten beide Konzepte, dass ein Mensch im Abgleich mit den Umweltanforderungen als mehr oder weniger fähig zu bestimmten Aktivitäten beschrieben werden kann, bzw. mit seinen Fähigkeiten mehr oder weniger gut zu bestimmten Arbeitsanforderungen und Arbeitsplätzen passt. Nach diesem Verständnis ist es nicht möglich, einen generell guten Arbeitsplatz zu definieren, sondern nur einen guten Arbeitsplatz für Mitarbeitende mit bestimmten Merkmalen und Fähigkeiten. Dieses Modell liegt auch den offiziellen medizinischen Arbeitsunfähigkeitsrichtlinien zugrunde, nach denen Arbeitsfähigkeit von Erwerbstätigen mit Gesundheitsproblemen befundet wird (GBA 2016). Das relationale Konzept der Arbeitsfähigkeit zu verstehen ist nicht nur für (Betriebs) Ärzt*innen von Belang, sondern auch für Personaler oder Führungskräfte. Letztere sind diejenigen, die die Arbeitsanforderungen kennen, definieren, und die passenden Mitarbeitenden an die jeweiligen Arbeitsplätze setzen, also den Person-Job-Fit in der betrieblichen Praxis herstellen sollen.

Das relationale Modell (Person-Job-Fit, ICF-Gesundheitsmodell) ermöglicht im Kontext der Arbeitsfähigkeitsbestimmung eine umfassende Betrachtung des Menschen mit seinen Eigenschaften eingebettet in seinen jeweiligen Lebenskontext. Neben dem körperlichen Zustand werden auch die damit einhergehenden Fähigkeiten oder -beeinträchtigungen erfasst. Ein Mensch mit einem gebrochenen Arm (Gesundheitsstörung), ist nicht in der Lage den Arm zu heben (Fähigkeitsbeeinträchtigung). Als Kellner*in (Kontextanforderung) wäre man damit vorübergehend arbeitsunfähig. Als Fußballtrainer*in am Spielfeldrand wäre man arbeitsfähig.

Eine Gesundheitsstörung an sich ist also nicht gleichzusetzen mit einer Arbeitsunfähigkeit. Arbeitsfähigkeit kann nur beurteilt werden, wenn bekannt ist, welche Aktivitäten und Fähigkeiten genau die Arbeit erfordert.

Etwas schwieriger als bei einem Armbruch ist es zu bestimmen, welche Fähigkeitsbeeinträchtigungen bei einem Arbeitstätigen entstehen können, der Ängste, Stimmungsstörungen oder Interaktionsstörungen hat. Dass derartige psychische Problemlagen hochrelevant sind, zeigen epidemiologische Studien nach denen - über Jahrzehnte hinweg stabil - etwa 25-30\% der Allgemein- und Erwerbspersonen von einer psychischen Erkrankung betroffen sind (Wittchen et al. 2011; Stansfeld et al. 2008; Gjesdal et al. 2008). Zudem lässt sich seit etwa zehn Jahren ein Trend zunehmender Arbeitsunfähigkeiten und Erwerbsunfähigkeitsberentungen ,wegen psychischer Erkrankungen“ beobachten (DRV 2019). Dies liegt nicht daran, dass Arbeit krank macht. Die epidemiologischen Erkrankungszahlen sind über die Jahre hinweg gleich geblieben (Jacobi und Linden 2018); es werden jedoch zunehmend psychische Fähigkei- 
ten gefordert (BMAS 2017). Menschen mit psychischen Erkrankungen können diese ggf. schwerer bewältigen. Eine Krankheit allein begründet somit keine Arbeitsunfähigkeit. Ausschlaggebend sind das Fähigkeitsprofil und die Passung von Anforderungen und Fähigkeiten.

Zur Erfassung von Fähigkeiten gibt es viele Messinstrumente (z.B. ADL- oder IADL-Skalen, Lawton und Brody 1968; GAS, Endicott et al. 1976; WHODAS 2.0, WHO 2004; ICF-Core-Sets, Cieza et al. 2004; Übersicht in Linden et al. 2015). Diese sind jedoch entweder sehr global, erfassen nur einen einschränkten Anwendungsbereich oder differenzieren nicht ausreichend zwischen körperlichem Zustand/Symptomen einerseits und Fähigkeiten und Teilhabe andererseits (Buchholz et al. 2015). Je nach Anforderungen der Umwelt (Kontext) haben Fähigkeiten(beeinträchtigungen) eine sehr unterschiedliche Teilhaberelevanz ${ }^{1}$ bzw. sozialmedizinische Bedeutung: Rezeptionist*innen benötigen andere Fähigkeiten als Automechaniker*innen oder Buchhalter*innen.

\section{Das Fähigkeitenkonzept nach Mini-ICF- APP}

Das Fähigkeitskonzept des Mini-ICF-APP basiert auf dem funktionalen Gesundheitsmodell der Internationalen Klassifikation der Funktionsfähigkeit, Behinderung und Gesundheit (ICF, WHO 2001). In der ICF ist die Komponente der „Fähigkeiten und Aktivitäten“ als eine der wesentlichen Säulen in der Beschreibung von menschlichen (Gesundheits)Zuständen beschrieben und klassifiziert. Fähigkeiten ermöglichen Alltags- und Arbeitsaktivitäten und sind die Voraussetzung dafür, dass Menschen in ihrer Lebensumwelt teilhaben und ein erfolgreiches Leben führen können.

Die Mini-ICF-APP-Fähigkeitskonzept umfasst 13 psychische Fähigkeiten (Soft Skills). Das Assessment umfasst Instrumente zur Beschreibung von psychischen Fähigkeiten und Fähigkeitsbeeinträchtigungen von Personen sowie Fähigkeitsanforderungen an Arbeitsplätzen (Linden et al. 2009, 2015; Muschalla 2020).

Die 13 Fähigkeitsdimensionen des Mini-ICF-APP liefern außerdem auch eine Art Gliederungsmatrix für einen psychischen Fähigkeitsbefund, oder für einen Abgleich von Fähigkeitsprofil der Person und Anforderungsprofil des Arbeitsplatzes. Die 13 Fähigkeitsdimensionen im Mini-ICFAPP-Konzept sind:

- Fähigkeit zur Anpassung an Regeln und Routinen

- Fähigkeit zur Planung und Strukturierung von Aufgaben

\footnotetext{
1 Teilhabe: Eingebundensein in eine Lebensumwelt. Fähigkeitsbeeinträchtigungen führen regelhaft zu Problemen in der Bewältigung von Aufgaben und der Erfüllung sozialer Rollen. Sie sind also relevant für die Teilhabe in dem Sinne, dass sie sie beeinträchtigen können.
}

- Flexibilität und Umstellungsfähigkeit

- Kompetenz- und Wissensanwendung

- Entscheidungs- und Urteilsfähigkeit

- Proaktivität und Spontanaktivitäten

- Widerstands- und Durchhaltefähigkeit

- Selbstbehauptungsfähigkeit

- Konversation und Kontaktfähigkeit zu Dritten

- Gruppenfähigkeit

- Fähigkeit zu engen dyadischen Beziehungen

- Fähigkeit zur Selbstpflege und Selbstversorgung

- Mobilität und Verkehrsfähigkeit

Das Fähigkeitskonzept ist international validiert, übersetzt und im deutschsprachigen sozialmedizinischen Kontext der Arbeitsfähigkeitsbeschreibung ein Standardinstrument geworden. Es wird in Leitlinien zur Verwendung empfohlen (Linden et al. 2009, 2015; DRV 2012; SGVP 2016; AWMF 2019; Molodynski et al. 2013; Balestrieri et al. 2013; DGPM/DKPM 2020).

Es handelt sich beim Mini-ICF-APP nicht um einen „Test“", sondern um ein System zur Dokumentation, Objektivierung und differenzierten Beschreibung von Fähigkeiten einer Person oder Fähigkeitsanforderungen an Arbeitsplätzen. Grundlage der Mini-ICF-APP Instrumentenfamilie sind die oben genannten 13 Fähigkeitsdimensionen. Sie werden anhand verhaltensnaher Ankerdefinitionen ausführlich beschrieben (Anhang A; Linden et al. 2009, 2015) und wurden an inhaltlich angrenzenden Aktivitäten-Interviews und -skalen validiert (Endicott und Nee 1997; Wiersma et al. 1990; Linden et al. 2010, 2009).

Im Folgenden werden die drei Operationalisierungen des Mini-ICF-APP (Fremdrating, Selbstrating und Arbeitsanforderungsrating) mit ihren Items (Anhänge A, B und C) und hinsichtlich der jeweils bedeutsamen Anwendungsaspekte vorgestellt.

\subsection{Fremdbeurteilung von Fähigkeiten(beeinträchtigungen) mit dem Mini-ICF-APP}

Vor Beginn der Fähigkeitenexploration nach dem MiniICF-APP-Fähigkeitenbogen ist der sogenannte Referenzkontext festzulegen (d.h. die Fähigkeits-Anforderungen), auf den sich die Beurteilung beziehen soll. Wenn bspw. eine Betriebsärztin oder -psychologin eines Klinikums erfährt, dass ein wiedereinzugliedernder Beschäftigter als „Gesundheits- und Krankenpfleger" tätig ist, weiß man noch nichts darüber, welche Aktivitäten am Arbeitsplatz konkret von dem Beschäftigten verlangt wird. Je nachdem, ob er im Blutspendedienst, in der Notaufnahme, oder auf der psychiatrischen Station arbeiten, kann er sehr verschiedene Fähigkeitsanforderungen haben: hohe Regelroutine, oder ho- 
he Flexibilität, oder ausgeprägte interaktionelle Fähigkeiten (Kontaktfähigkeit, Selbstbehauptung, Gruppenfähigkeit).

Die Fähigkeitsanforderungen müssen konkret erfragt werden, z. B. „Können Sie bitte erzählen, wie ein üblicher Tag bei Ihrer Arbeit aussieht? Was müssen Sie konkret tun, wenn Sie morgens um 7 Uhr zur Arbeit kommen?“. Ziel ist es, eine möglichst genaue Auflistung des geforderten Aktivitätenspektrums zu bekommen. Diese können den entsprechenden Fähigkeitsdimensionen des Mini-ICF-APP zugeordnet werden.

Im nächsten Schritt ist zu explorieren, ob es mit der Bewältigung der einzelnen Aufgaben Probleme gibt, und falls ja, wie ausgeprägt diese sind: Sind sie nur für den Beschäftigten merkbar im Sinne von es fällt ihm schwer (leichte Beeinträchtigung), oder sind sie auch für andere Kolleg*innen oder Vorgesetzten beobachtbar und ggf. ärgerlich (mäßige Beeinträchtigung), oder führen sie dazu, dass regelmäBig jemand einspringen und unterstützen oder die Aufgabe komplett abnehmen muss (schwere Beeinträchtigung)? Dabei gilt, dass der Anforderungsgrad auch den Grad der Beeinträchtigung mitbestimmt: Für Stewardessen, die Kolleg*innen ablösen sollen, ist zehnminütiges zu-Spät-Kommen (Fähigkeitsproblem: Anpassung an Regeln und Routinen) eine schwere Beeinträchtigung und führt ggf. zur Entlassung. Bei Büromitarbeiter*innen fällt es ggf. kaum auf, ob sie ein paar Minuten später am Schreibtisch sitzen, allenfalls haben sie möglicherweise selbst ein schlechtes Gewissen, weil sie sich vorgenommen haben früher ins Büro zu kommen (leichte Beeinträchtigung).

Je nach Anforderungsgrad (Pünktlichkeit bei Stewardess versus Büromitarbeiter*in) fällt das gleiche Fähigkeitsproblem an einem Arbeitsplatz unterschiedlich stark ins Gewicht. Wichtig für die valide Anwendung des Mini-ICFAPP Fremdratings ist also, die Fähigkeitsanforderungen sehr konkret zu kennen bzw. zu definieren.

In der betrieblichen Praxis relevant sind Beeinträchtigungen, die ständigen Ärger im Betriebsablauf verursachen und ein regelmäßiges Unterstützen Dritter erfordern (schwere Beeinträchtigung), um die vorgesehenen Aufgaben zu erledigen. Da es auf dem allgemeinen Arbeitsmarkt in der Regel wenig Freiheitsgrade gibt, für die Tätigkeiten einzelner Mitarbeitender ständig eine zweite Person zum Einspringen zur Verfügung zu stellen, ist in vielen Fällen eine Arbeitsunfähigkeit gegeben, wenn Fähigkeiten ständig in einer unterstützungsbedürftigen Weise, d.h. schwer beeinträchtigt sind.

Wenn eine Fähigkeitsbeeinträchtigung vorliegt ist zu klären, ob die Beeinträchtigungen krankheitsbedingt sind oder andere Gründe haben (z.B. Qualifikations- oder Trainingsdefizit oder konstitutionell bedingte Fähigkeitsschwäche). Hierzu braucht es eine/n Betriebsärzt*in. Fehlt es beispielsweise an ausreichender beruflicher Qualifikation oder Willensanstrengung, würde man keine „krankheitsbedingte“
Fähigkeitsbeeinträchtigung attestieren. Die festgestellten Abweichungen vom Soll wären dann nicht sozialmedizinisch von Bedeutung, sondern arbeitsrechtlich.

Fähigkeitsanforderungen zu definieren und Fähigkeitsprobleme zu erkennen und verhaltensnah zu beschreiben, kann durch Führungskräfte und das Arbeitsteam geschehen. Hierzu sind qualitative Beobachtungen hilfreich sowie ggf. ein Abgleich der Fähigkeitsanforderungen, die die Beschäftigten an dem Arbeitsplatz wahrnehmen (Anhang C) und der Fähigkeitsprofil-Selbstratings von Mitarbeitenden (Anhang B).

Eine Fremdbeurteilung und Beschreibung von Arbeitsfähigkeit im sozialmedizinischen Kontext ist jedoch Aufgabe von Ärzt*innen, ggf. unterstützt von sozialmedizinisch geschulten (Betriebs-)Psychotherapeuten*innen.

\subsection{Fähigkeiten-Selbsteinschätzung mit dem Mini- ICF-APP-S (Selbstrating)}

Im Bereich der Fähigkeitsdiagnostik kommen auch Selbstbeurteilungen zum Einsatz. Beispiele sind der ICF-AT50-Psych („Aktivitäten und Teilhabe“; Nosper 2008), das Selbstrating zum WHO-DAS-2.0 (WHO 2004) oder der ICFPsych A\&P (Brütt et al. 2015).

Mit Selbsteinschätzungen können Informationen über subjektiv wahrgenommene und erlebte Stärken und Schwächen ergänzend zur Fremdbeurteilung differenziert erfasst werden. Sie lassen sich ökonomisch einsetzen, um einen Einblick in die Selbstsicht von Personen auf ihr Fähigkeitsniveau und -profil zu erhalten, ohne dabei nach Gesundheitsbeeinträchtigungen fragen zu müssen. Auch lassen sich Veränderungsprozesse zwischen verschiedenen Messzeitpunkten abbilden, oder Interventionsentscheidungen unterstützen (Laireiter 2005).

Um ergänzend zur Fremdbeurteilung auch die Selbstschätzung von Menschen zu ihren eigenen wahrgenommenen Fähigkeiten erheben zu können, wurde der Selbstbeurteilungsbogen Mini-ICF-APP-S entwickelt und in einer Untersuchung von Patient*innen mit psychischen Erkrankungen sowie einer Allgemeinbevölkerungsstichprobe evaluiert (Linden et al. 2018). Er umfasst dieselben 13 Fähigkeiten (,Soft-Skills“) des Mini-ICF-APP. Die Proband*innen können auf einer Skala ihre jeweiligen Fähigkeiten einschätzen von ,, $0=$ das ist eindeutig eine Stärke von mir“ über , $3=$ das geht schon irgendwie“ und „4= das klappt nicht immer" bis hin $\mathrm{zu},, 7=$ das kann ich gar nicht.“ (Anhang B).

\subsection{Fähigkeitsorientierte Arbeitsplatzbeschreibung mit dem Mini-ICF-APP-W (Work)}

Seit Jahrzehnten sind die Prävalenzen psychischer Erkrankungen stabil (Wittchen et al. 2011; Jacobi und Linden 2018; Zielke 2017). Die Sichtweise, dass Arbeit krank ma- 
che, verhindert aber, dass das eigentliche Problem der modernen Arbeitswelt erkannt und angegangen wird: der zunehmend problematische Person-Job-Fit (Jacobi und Linden 2018) für Beschäftigte mit psychischen Erkrankungen. Psychische Erkrankungen sind chronische Erkrankungen und Volkskrankheiten, etwa 25-30\% der Menschen leiden an einer psychischen Erkrankung (Stansfeld et al. 2008; Wittchen et al. 2011). Ein Großteil von ihnen ist berufstätig: Bspw. hatten von 307 Hausarztpatient*innen mit chronischen psychischen Erkrankungen $66 \%$ einen Arbeitsplatz (Linden und Muschalla 2018). Psychische Erkrankungen am Arbeitsplatz sind daher ein nicht zu vernachlässigendes Phänomen. Die moderne Arbeitswelt macht allerdings nicht krank, wie zuweilen debattiert wird, sondern stellt heute mehr als früher Anforderungen an kognitive und interpersonelle Leistungen (BMAS 2017). Die zu erfüllen kann Menschen mit psychischen Erkrankungen Schwierigkeiten bereiten (Meier-Credner und Muschalla 2019; Jacobi und Linden 2018). Menschen mit psychischen Erkrankungen fallen daher vermehrt mit Arbeitsunfähigkeit auf (GBA 2016) oder ganz aus der Arbeitswelt heraus. In der Konsequenz gilt, dass wieder mehr sogenannte Toleranzarbeitsplätze benötigt werden. Im Sinne der ICF (WHO 2001) und des Person-Job-Fit bedeutet dies, dass die Arbeitsanforderungen so eingerichtet werden sollten, dass sie zu den menschlichen Fähigkeiten passen und Freiheitsgrade ermöglichen. Auch das Arbeitsschutzgesetz ( $\$ 5$ ArbSchG 2013) fordert von Arbeitgeber*innen, psychisches Gefährdungspotential an Arbeitsplätzen zu minimieren. Dies lässt sich durch die Herstellung fähigkeitsgerechter Arbeitsplätze erreichen. Der Arbeitsplatz muss zu den Fähigkeiten der Mitarbeitenden passen. Um einen Person-Job-Fit prüfen zu können braucht es eine Beschreibung von Mitarbeiterfähigkeiten und Arbeitsanforderungen entsprechend eines einheitlichen Konzepts. Dies wird mittels der Mini-ICF-Instrumentenfamilie gewährleistet.

Analog zum Fähigkeiten-Selbst- und Fremdrating wurde dementsprechend das Mini-ICF-APP-W für die Beschreibung von Arbeits-Fähigkeits-Anforderungen entwickelt (Muschalla 2018a, 2018b; Anhang C). Mit dem MiniICF-APP-W(ork)-Rating kann auf einen konkreten Arbeitskontext bezogen beurteilt werden, in welchem Maße eine Tätigkeit verschiedene psychische Fähigkeiten erfordert. Eine Validierung des Mini-ICF-APP-W wurde bei 166 chronisch kranken Erwerbsaltrigen durchgeführt (Muschalla 2018a, 2018b). Die Inter-Rater-Reliabilität lag bei $r=0,63-0,91$ (Muschalla 2018a). Bei Patient*innen, die eine psychische Problematik hatten, wurde ein Abgleich von Fähigkeitsanforderungen (Mini-ICF-APP-W) und Fähigkeitsprofil der Person (Mini-ICF-APP) vorgenommen. Bei Patient*innen mit negativer erwerbsbezogener Prognose, d.h. voraussichtlich dauerhafter Unfähigkeit ihre zuletzt ausgeübte Tätigkeit wieder aufzunehmen, bestand in den
Dimensionen Flexibilität, Durchhaltefähigkeit sowie den interaktionellen Fähigkeiten (Selbstbehauptung, Kontakt-, und Gruppenfähigkeit) ein Mismatch: Die Fähigkeitsausprägungen der Personen waren gering, bei gleichzeitig höher erlebten Arbeitsanforderungen.

\section{Diskussion}

\subsection{Fremdbeurteilung und Selbstbeurteilung von Fähigkeiten im Vergleich}

Bei sozialmedizinischen Beurteilungen der Arbeitsfähigkeit werden besondere Kompetenzen vom Beurteiler verlangt. Kompetenz zur Beschreibung von Arbeits- und Leistungsfähigkeit/sdefiziten bei Gesundheitsproblemen haben Ärzt*innen und sozialmedizinisch geschulte Psychotherapeut*innen. In die Arbeitsfähigkeitsbeurteilung fließen sowohl die Selbstaussagen der Patient*innen aber auch die Verhaltensbeobachtung und explorierte Daten durch die Untersuchenden sowie Vorbefunde und Behandlungsverläufe mit ein.

Bei dieser klinischen Urteilsbildung mögliche Fehlerquellen (Halo-Effekt: Generalisierung von einer Beeinträchtigung auf andere Bereiche, unterschiedliches Verständnis oder Schwerpunktsetzung in der Exploration oder Beschreibung von Anforderungen oder Beeinträchtigungen) können aber durch intensive Trainings in der Anwendung von Fremdbeurteilungsverfahren verringert und dadurch gute Interrater-Reliabilitäten erzielt werden. Beim Mini-ICF-APP wurde die Interrater-Reliabilität von 0,7 (ungeschulte Rater) bis auf 0,9 (trainierte Rater) verbessert (Linden et al. 2009).

Ein entscheidender Validitätshinweis aus der ersten Untersuchung mit dem Fähigkeiten-Selbstrating (Mini-ICFAPP-S, Linden et al. 2018) ist, dass sich Patient*innen, die im ärztlichen Urteil bei Entlassung arbeitsfähig sind oder nicht, bereits bei der stationären Aufnahme hinsichtlich ihres subjektiv eingeschätzten Fähigkeitsniveaus unterscheiden. Ältere schätzen ihr Fähigkeitsniveau besser ein als jüngere, z.B. in der Proaktivität, Planungsfähigkeit, Selbstbehauptungsfähigkeit, und Durchhaltefähigkeit. Bei den untersuchten Rehabilitationspatient*innen lag der Altersdurchschnitt bei 50 Jahren, es geht also um die erfahreneren Arbeitnehmer*innen. Die Daten entsprechen auch langjährigen klinischen Beobachtungen, wonach reifere Personen mehr Übersicht zeigen als jüngere Menschen sowie mehr Durchhaltevermögen oder auch eine höhere Sozialkompetenz.

Der Kontextbezug bleibt bei der Selbstbeurteilung im Gegensatz zum Fremdrating nach außen hin unklar. Insgesamt scheint es sich eher um ein Rating der Zufriedenheit mit den eigenen Fähigkeiten zu handeln, als um eine objek- 
tive Beschreibung seines Zustandes (Linden et al. 2018). Trotz der genannten Einschränkungen in der Validität von Selbstbeurteilungen liefert das Mini-ICF-APP-S wichtige Informationen darüber, ob und wo die Proband*innen Schwierigkeiten erlebten. Selbst- und Fremdeinschätzung liefern unterschiedliche Perspektiven und können daher ergänzend zum Einsatz kommen. Mögliche Diskrepanzen zwischen der Selbst- und Fremdbeurteilung können in Gesprächen mit den Beteiligten (Mitarbeitenden, Führungskraft, Betriebsärzt*in) geklärt werden und ggf. passgenaue Lösungen für die jeweilige Arbeitsplatzkonstellation erarbeitet werden.

\subsection{Interventionsmöglichkeiten auf Basis von Fähigkeiten-Diagnostik}

In der betrieblichen Praxis interessiert weniger ein Mittelwert der 13 Fähigkeiten, sondern vielmehr eine konkrete verhaltensnahe Beschreibung einzelner relevanter Fähigkeiten(beeinträchtigungen) sowie auch Fähigkeitsreserven Mitarbeitender. So kann beispielsweise eine Beeinträchtigung nur einer Fähigkeit schlimmstenfalls bereits eine Arbeitsunfähigkeit nach sich ziehen (z.B. Mobilität bei Mitarbeitenden beim städtischen Ordnungsamt). Andererseits ist auch die Berücksichtigung intakter Fähigkeiten praktisch relevant, wenn diese kompensatorisch genutzt werden können (z.B. die Fähigkeit Kolleg*innen dazu zu bringen, Wege außer Haus für die eigene Person zu übernehmen, während man den entsprechenden Kollegen*innen Innendienstaufgaben abnimmt).

Die Beschreibung von Stärken und Beeinträchtigungen und somit einem Fähigkeitenprofil ermöglicht es, Passung und Nichtpassung von Fähigkeiten und Anforderungen zu beschreiben und gezielte Interventionen zur Optimierung der Arbeits- und Leistungsfähigkeit zu finden. Beispiele sind zum einen Fähigkeitstrainings (Muschalla 2014) wie Soziales Kompetenztraining (Hinsch und Pfingsten 2002; Löffler et al. 2012; Smith 2003) bei Beeinträchtigungen der Selbstbehauptungs-, Kontakt- oder Gruppenfähigkeit, oder Problemlösetraining (D'Zurilla und Goldfried 1971; Kaluza 2004) bei Problemen in der Fähigkeit zur Planung und Strukturierung von Aufgaben oder der Entscheidungsund Urteilsfähigkeit.

Neben Fähigkeitentrainings können auch (vorübergehend) Kontextänderungen zur Besserung oder Kompensation von Fähigkeitseinschränkungen eingesetzt werden. Dazu gehören beispielsweise eine entsprechende Gestaltung des Arbeitsplatzes (z. B. Anschaffung einer Lesebrille, eines speziellen Schreibtischstuhls, die Verringerung des Arbeitsweges durch Versetzung in eine andere Dienststelle, Flexibilisierung der Arbeitszeit), Veränderung der Art der Aufgaben, so dass sie zu den Fähigkeitsstärken der Mitarbeitenden passen (z.B. der Kontaktfreudige mit
Aufmerksamkeitsstörung macht Kundengespräche statt Buchhaltung), Maßnahmen im Rahmen des betrieblichen Eingliederungsmanagements (BEM, § 167 SGB IX) oder Leistungen zur Teilhabe am Arbeitsleben (LTA, § 49 SGB IX).

Bei Fällen von krankheitsbedingten Fähigkeitsdefiziten ist immer (betriebs)ärztliche Expertise hinzuzuziehen, damit die passende Maßnahme zum Arbeitsfähigkeitserhalt ausgewählt werden kann.

\subsection{Fazit für die Praxis}

Mit dem Fähigkeitenkonzept nach Mini-ICF-APP kann das Fähigkeitsprofil eines Menschen mit dem Profil der Fähigkeitsanforderungen verglichen werden. Wenn am Arbeitsplatz Fähigkeiten verlangt werden, die eine Person nicht erbringen kann, dann liegt ein Problem mit dem „PersonJob-Fit" vor. Darauf gibt es drei Reaktionen (Linden et al. 2015):

a) die Wiederherstellung der unzureichenden Fähigkeiten (bei veränderbarem Fähigkeitsniveau, mittels Training; Muschalla 2014), und damit (Wieder-)Erreichung des „Person-Job-Fit"

b) die Entpflichtung von der Arbeit, z.B. durch ein Arbeitsunfähigkeitsattest, und

c) die Herstellung eines passenden Arbeitsplatzes, d.h. einen „Person-Job-Fit“ (bei nicht veränderbarem Fähigkeitsniveau).

\section{Anhang A}

\section{Fähigkeiten-Fremdbeurteilung: Mini-ICF-APP (zitiert und modifiziert nach Linden et al. 2009)}

Das Ausmaß der Fähigkeitsbeeinträchtigungen ist nach folgender Abstufung zu quantifizieren:

0: keine Beeinträchtigung: Der/die Proband*in entspricht den Normerwartungen bezüglich seiner/ihrer Referenzgruppe.

1: leicht ausgeprägte Beeinträchtigung: Es bestehen einige leichtere Schwierigkeiten oder Probleme, die beschriebenen Fähigkeiten/Aktivitäten auszuüben. Es resultieren daraus keine wesentlichen negativen Konsequenzen.

2: mäßig ausgeprägte Beeinträchtigung: Im Vergleich zur Referenzgruppe bestehen deutliche Probleme, die beschriebenen Fähigkeiten/Aktivitäten auszuüben. Dies hat negative Auswirkungen bzw. negative Konsequenzen für den Probanden oder andere.

3: erhebliche ausgeprägte Beeinträchtigung: Der/die Proband*in ist wesentlich eingeschränkt in der Ausübung der beschriebenen Fähigkeiten/Aktivitäten. Der/die Proband*in kann Rollenerwartungen in wesentlichen Teilen 
nicht mehr gerecht werden. Der/die Proband*in benötigt teilweise Unterstützung von Dritten.

4: voll ausgeprägte Beeinträchtigung: Der/die Proband*in ist nicht in der Lage, die beschriebenen Fähig-
keiten/Aktivitäten auszuüben. Der/die Proband*in muss entpflichtet werden, und die Aktivitäten müssen durch Dritte übernommen werden.
Abb. A.1 Durch den aktuellen Gesundheitszustand liegen Beeinträchtigungen vor bezüglich

\begin{tabular}{|c|c|c|}
\hline $\begin{array}{l}\text { Kontext: } \\
\text { O Arbeitsplatz: }\end{array}$ & & einträchtigung \\
\hline $\begin{array}{l}O \text { Berufsfeld: } \\
O \text { allgemeiner Arbeitsmarkt } \\
O \text { anderes: }\end{array}$ & Grad & $\begin{array}{l}\text { Konkrete } \\
\text { Beschreibung der } \\
\text { Beeinträchtigung }\end{array}$ \\
\hline $\begin{array}{l}\text { 1. Anpassung an Regeln und Routinen } \\
\text { Fähigkeit, sich an Regeln zu halten, Termine } \\
\text { verabredungsgemäß wahrzunehmen und sich in } \\
\text { Organisationsabläufe einzufügen. Dies beinhaltet bspw. die } \\
\text { Erfüllung von täglichen Routineabläufen, Einhalten von } \\
\text { Verabredungen, pünktliches Erscheinen, Einhaltung von } \\
\text { Verfahrensvorschriften. }\end{array}$ & & \\
\hline $\begin{array}{l}\text { 2. Planung und Strukturierung von Aufgaben } \\
\text { Fähigkeit, den Tag und/oder anstehende Aufgaben zu planen } \\
\text { und zu strukturieren, d. h. angemessene Zeit für Aktivitäten } \\
\text { (Arbeit, Haushaltsführung, Erholung und andere Tages- und } \\
\text { Freizeitaktivitäten) aufzuwenden, die Reihenfolge der } \\
\text { Arbeitsabläufe sinnvoll zu strukturieren, diese wie geplant } \\
\text { durchzuführen und zu beenden. }\end{array}$ & & \\
\hline $\begin{array}{l}\text { 3. Flexibilität und Umstellungsfähigkeit } \\
\text { Fähigkeit, sich im Verhalten, Denken und Erleben wechselnden } \\
\text { Situationen anzupassen, d. h. je nach Situation und } \\
\text { Anforderung unterschiedliche Verhaltensweisen zu zeigen. } \\
\text { Dies kann Veränderungen in den Arbeitsanforderungen, } \\
\text { kurzfristige Zeitveränderungen, räumliche Veränderungen, } \\
\text { neue Sozialpartner oder auch die Übertragung neuer Aufgaben } \\
\text { betreffen. }\end{array}$ & & \\
\hline $\begin{array}{l}\text { 4. Kompetenz- und Wissensanwendung } \\
\text { Fähigkeit zur Anwendung fachlicher Kompetenzen, d. h. } \\
\text { beruflich, ausbildungsspezifisch oder auf Grund der } \\
\text { Lebenserfahrung. Fähigkeit, Fach- und Lebenswissen oder } \\
\text { Kompetenzen gemäß den situativen Rollenerwartungen } \\
\text { einzusetzen und unter Berücksichtigung des } \\
\text { Lebenshintergrunds zumutbare inhaltliche und fachliche } \\
\text { Anforderungen zu erfüllen. }\end{array}$ & & \\
\hline $\begin{array}{l}\text { 5. Entscheidungs- und Urteilsfähigkeit } \\
\text { Fähigkeit, kontextbezogen und nachvollziehbar Entscheidungen } \\
\text { zu fällen oder Urteile abzugeben. Fähigkeit, Sachverhalte } \\
\text { differenziert und kontextbezogen auffassen, daraus die } \\
\text { angemessenen Schlussfolgerungen und Konsequenzen ziehen } \\
\text { und dies in erforderliche Entscheidungen umsetzen zu können. }\end{array}$ & & \\
\hline $\begin{array}{l}\text { P. Proaktivität und Spontanaktivitäten } \\
\text { Fähigkeit, außerhalb vorgegebener beruflicher oder sozialer } \\
\text { Pflichten eigeninitiativ Spontanaktivitäten zu initiieren. } \\
\text { Beurteilt werden Aktivitäten, bei denen die Proband*innen } \\
\text { selbst aktiv und initiativ werden müssen und die nicht } \\
\text { beispielsweise durch eine Berufsrolle oder sonstige Pflichten } \\
\text { aufgezwungen werden. Dazu gehören zum einen Aktivitäten } \\
\text { des täglichen Lebens wie häusliche Aktivitäten, z. B. die } \\
\text { Beschaffung von Waren- und Dienstleistungen des täglichen } \\
\text { Bedarfs, die Zubereitung von Mahlzeiten, die Pflege von } \\
\text { Wohnung, Haus und Haushaltsgegenständen, die Versorgung } \\
\text { von Pflanzen oder Haustieren. Dazu gehören des Weiteren } \\
\text { kreative oder rekreative Aktivitäten, z. B. Hobbys, der Besuch } \\
\text { von kulturellen Veranstaltungen, Erholungsaktivitäten, Sport } \\
\text { oder künstlerische Aktivitäten. }\end{array}$ & & \\
\hline
\end{tabular}


Abb. A.2 (Fortsetzung)

\begin{tabular}{|c|c|}
\hline 7. & $\begin{array}{l}\text { Widerstands- und Durchhaltefähigkeit } \\
\text { Fähigkeit, hinreichend ausdauernd und während der } \\
\text { üblicherweise erwarteten Zeit an einer Tätigkeit (im Beruf oder } \\
\text { bei sonstigen Aufgaben) bleiben und ein durchgehendes } \\
\text { Leistungsniveau aufrechterhalten zu können. }\end{array}$ \\
\hline 8. & $\begin{array}{l}\text { Selbstbehauptungsfähigkeit } \\
\text { Fähigkeit, in sozialen Kontakten oder auch Konfliktsituationen } \\
\text { ohne beeinträchtigende Befangenheit bestehen und für seine } \\
\text { Überzeugungen einstehen zu können, ohne dabei soziale } \\
\text { Normen zu verletzen. }\end{array}$ \\
\hline 9. & $\begin{array}{l}\text { Konversation und Kontaktfähigkeit zu Dritten } \\
\text { Fähigkeit, unmittelbare informelle soziale Kontakte mit } \\
\text { anderen Menschen aufnehmen zu können, wie bei } \\
\text { Begegnungen mit Kolleg*innen, Nachbar*innen oder } \\
\text { Bekannten und mit diesen angemessen interagieren zu können, } \\
\text { wozu auch Rücksichtnahme, Wertschätzung des Gegenübers } \\
\text { oder die Fähigkeit, Gespräche zu führen gehören. Dazu gehört } \\
\text { die Fähigkeit des Probanden, unverbindlich kommunizieren zu } \\
\text { können }\end{array}$ \\
\hline 10. & $\begin{array}{l}\text { Gruppenfähigkeit } \\
\text { Fähigkeit, sich in Gruppen einfügen, die expliziten oder } \\
\text { informellen Regeln der Gruppe durchschauen und sich darauf }\end{array}$ \\
\hline & $\begin{array}{l}\text { einstellen zu können. Die Beurteilung bezieht sich auf das } \\
\text { Verhalten der Proband*innen in Gruppensituationen bzw. sihre } \\
\text { Fähigkeiten zur öffentlichen Präsentation. Dazu gehören } \\
\text { Kleingruppen wie das Arbeitsteam, der Verein oder } \\
\text { Großgruppen wie die Firma, eine politische Gruppierung oder } \\
\text { die Kirche. }\end{array}$ \\
\hline 11. & $\begin{array}{l}\text { Fähigkeit zu engen dyadischen Beziehungen } \\
\text { Fähigkeit, enge und ggf. intime Beziehungen zu vertrauten } \\
\text { Menschen oder in der Familie aufnehmen und aufrechterhalten } \\
\text { zu können. Beurteilt wird die Fähigkeit, enge emotionale } \\
\text { Zuwendung zu geben und zu empfangen und mit den anderen } \\
\text { Rollenerwartungen und dem beruflichen Umfeld befriedigend } \\
\text { abzustimmen. }\end{array}$ \\
\hline 12. & $\begin{array}{l}\text { Selbstpflege und Selbstversorgung } \\
\text { Fähigkeit, zur Selbstpflege und -versorgung, also die Fähigkeit, } \\
\text { sich zu waschen, Haare oder Zähne zu pflegen, sich dem Anlass } \\
\text { oder der Jahreszeit entsprechend zu kleiden, sich adäquat zu } \\
\text { ernähren, auf Erholung zu achten, die gesundheitlichen } \\
\text { Bedürfnisse seines Körpers wahrzunehmen und darauf } \\
\text { angemessen zu reagieren. }\end{array}$ \\
\hline 13. & $\begin{array}{l}\text { Mobilität und Verkehrsfähigkeit } \\
\text { Fähigkeit, sich zu bewegen, alle üblichen geforderten } \\
\text { Bewegungen auszuüben, an verschiedene Orte zu gehen, sich in } \\
\text { verschiedene Situationen zu begeben und übliche } \\
\text { Transportmittel, wie Auto, Bus oder Flugzeug, zu benutzen. } \\
\text { Beurteilt wird, ob die Proband*innen ohne Probleme jeden } \\
\text { verkehrsüblichen Platz aufsuchen und jedes verkehrsübliche } \\
\text { Fortbewegungsmittel benutzen können. }\end{array}$ \\
\hline
\end{tabular}




\section{Anhang B}

\section{Fragebogen zur Fähigkeiten-Selbstbeschreibung: Mini-ICF-APP-S (zitiert nach Linden et al. 2018)}

Bitte beurteilen Sie im Folgenden, wie Sie mit den jeweiligen Anforderungen zurechtkommen. Bitte kreuzen Sie die Aussage an, die am ehesten auf Sie zutrifft.

\section{Anpassung an Regeln und Routinen}

Wie gut können Sie sich an Regeln halten, z. B. vorgeschriebene Arbeitsabläufe und Dienstwege einhalten, pünktlich zu Terminen erscheinen, Vereinbarungen einhalten?

\begin{tabular}{llllllll}
\hline Das ist & Da bin & Das & Das & Das & Deswegen & Da & Das \\
eindeu- & ich & kann & geht & klappt & gibt es & müs- & kann \\
tig eine & besser & ich & schon & nicht & schon & sen & ich \\
Stärke & als die & ganz & ir- & im- & mal Pro- & mir & gar \\
von mir & meisten & gut & gend- & mer & bleme & andere & nicht \\
& & & wie & & & helfen & \\
\hline
\end{tabular}

\section{Planung und Strukturierung von Aufgaben}

Wie gut sind Sie darin, Dinge zu planen? Also Ihren Tagesablauf zu organisieren, oder festzulegen, was Sie in einer Woche wann erledigen müssen, welche Erledigungen oder Arbeiten vorrangig sind? Können Sie angemessene Zeit für die einzelnen Dinge einplanen?

\begin{tabular}{|c|c|c|c|c|c|c|c|}
\hline $\begin{array}{l}\text { Das ist } \\
\text { eindeu- } \\
\text { tig eine } \\
\text { Stärke } \\
\text { von mir }\end{array}$ & $\begin{array}{l}\text { Da bin } \\
\text { ich } \\
\text { besser } \\
\text { als die } \\
\text { meisten }\end{array}$ & $\begin{array}{l}\text { Das } \\
\text { kann } \\
\text { ich } \\
\text { ganz } \\
\text { gut }\end{array}$ & $\begin{array}{l}\text { Das } \\
\text { geht } \\
\text { schon } \\
\text { ir- } \\
\text { gend- } \\
\text { wie }\end{array}$ & $\begin{array}{l}\text { Das } \\
\text { klappt } \\
\text { nicht } \\
\text { im- } \\
\text { mer }\end{array}$ & $\begin{array}{l}\text { Deswegen } \\
\text { gibt es } \\
\text { schon } \\
\text { mal Pro- } \\
\text { bleme }\end{array}$ & $\begin{array}{l}\mathrm{Da} \\
\text { müs- } \\
\text { sen } \\
\text { mir } \\
\text { andere } \\
\text { helfen }\end{array}$ & $\begin{array}{l}\text { Das } \\
\text { kann } \\
\text { ich } \\
\text { gar } \\
\text { nicht }\end{array}$ \\
\hline
\end{tabular}

\section{Flexibilität und Umstellungsfähigkeit}

Wie gut gelingt es Ihnen, mit Veränderungen zurechtzukommen, z. B. Änderung von Arbeitsabläufen, neues Büro, neue/r Kolleg*in, neue Computerprogramme oder Techniken? Wie geht es Ihnen mit kurzfristigen Terminveränderungen? Oder wenn Sie plötzlich irgendwo anders hinmüssen? Wie geht es Ihnen, wenn Sie bei einer Sache unterbrochen werden (z.B. Telefon klingelt) und wenn Sie spontan etwas Dazwischenkommendes erledigen sollen?

\begin{tabular}{|c|c|c|c|c|c|c|c|}
\hline $\begin{array}{l}\text { Das ist } \\
\text { eindeu- } \\
\text { tig eine } \\
\text { Stärke } \\
\text { von mir }\end{array}$ & $\begin{array}{l}\text { Da bin } \\
\text { ich } \\
\text { besser } \\
\text { als die } \\
\text { meisten }\end{array}$ & $\begin{array}{l}\text { Das } \\
\text { kann } \\
\text { ich } \\
\text { ganz } \\
\text { gut }\end{array}$ & $\begin{array}{l}\text { Das } \\
\text { geht } \\
\text { schon } \\
\text { ir- } \\
\text { gend- } \\
\text { wie }\end{array}$ & $\begin{array}{l}\text { Das } \\
\text { klappt } \\
\text { nicht } \\
\text { im- } \\
\text { mer }\end{array}$ & $\begin{array}{l}\text { Deswegen } \\
\text { gibt es } \\
\text { schon } \\
\text { mal Pro- } \\
\text { bleme }\end{array}$ & $\begin{array}{l}\mathrm{Da} \\
\text { müs- } \\
\text { sen } \\
\text { mir } \\
\text { andere } \\
\text { helfen }\end{array}$ & $\begin{array}{l}\text { Das } \\
\text { kann } \\
\text { ich } \\
\text { gar } \\
\text { nicht }\end{array}$ \\
\hline
\end{tabular}

\section{Kompetenz- und Wissensanwendung}

Können Sie Ihr Wissen und Ihre Erfahrung anwenden, wenn es gefragt ist, bspw. bei der Arbeit die Dinge so erledigen, wie es Ihrer Ausbildung oder Ihrem eigentlichen Fachwissen entspricht?

\begin{tabular}{|c|c|c|c|c|c|c|c|}
\hline $\begin{array}{l}\text { Das ist } \\
\text { eindeu- } \\
\text { tig eine } \\
\text { Stärke } \\
\text { von mir }\end{array}$ & $\begin{array}{l}\text { Da bin } \\
\text { ich } \\
\text { besser } \\
\text { als die } \\
\text { meisten }\end{array}$ & $\begin{array}{l}\text { Das } \\
\text { kann } \\
\text { ich } \\
\text { ganz } \\
\text { gut }\end{array}$ & $\begin{array}{l}\text { Das } \\
\text { geht } \\
\text { schon } \\
\text { ir- } \\
\text { gend- } \\
\text { wie }\end{array}$ & $\begin{array}{l}\text { Das } \\
\text { klappt } \\
\text { nicht } \\
\text { im- } \\
\text { mer }\end{array}$ & $\begin{array}{l}\text { Deswegen } \\
\text { gibt es } \\
\text { schon } \\
\text { mal Pro- } \\
\text { bleme }\end{array}$ & $\begin{array}{l}\mathrm{Da} \\
\text { müs- } \\
\text { sen } \\
\text { mir } \\
\text { andere } \\
\text { helfen }\end{array}$ & $\begin{array}{l}\text { Das } \\
\text { kann } \\
\text { ich } \\
\text { gar } \\
\text { nicht }\end{array}$ \\
\hline
\end{tabular}

\section{Entscheidungs- und Urteilsfähigkeit}

Wie gut kann man sich auf Ihre Einschätzungen, Ihren Rat oder Ihre Entscheidungen verlassen? Kommen Sie üblicherweise auf der Basis vorliegender Informationen zu Schlussfolgerungen, die andere auch für sinnvoll und überzeugend halten?

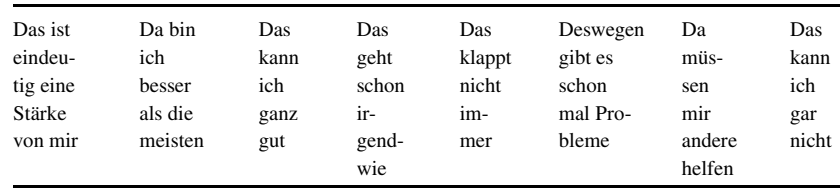

\section{Proaktivität und Spontanaktivitäten}

Wie gut gelingt es Ihnen in die Gänge zu kommen, eigene Initiativen zu ergreifen, von sich aus Dinge anzuregen und anzufangen, beispielsweise spontane Verabredungen, Freizeitaktivitäten, im Haushalt oder auch bei der Arbeit?

\begin{tabular}{|c|c|c|c|c|c|c|c|}
\hline $\begin{array}{l}\text { Das ist } \\
\text { eindeu- } \\
\text { tig eine } \\
\text { Stärke } \\
\text { von mir }\end{array}$ & $\begin{array}{l}\text { Da bin } \\
\text { ich } \\
\text { besser } \\
\text { als die } \\
\text { meisten }\end{array}$ & $\begin{array}{l}\text { Das } \\
\text { kann } \\
\text { ich } \\
\text { ganz } \\
\text { gut }\end{array}$ & $\begin{array}{l}\text { Das } \\
\text { geht } \\
\text { schon } \\
\text { ir- } \\
\text { gend- } \\
\text { wie }\end{array}$ & $\begin{array}{l}\text { Das } \\
\text { klappt } \\
\text { nicht } \\
\text { im- } \\
\text { mer }\end{array}$ & $\begin{array}{l}\text { Deswegen } \\
\text { gibt es } \\
\text { schon } \\
\text { mal Pro- } \\
\text { bleme }\end{array}$ & $\begin{array}{l}\mathrm{Da} \\
\text { müs- } \\
\text { sen } \\
\text { mir } \\
\text { andere } \\
\text { helfen }\end{array}$ & $\begin{array}{l}\text { Das } \\
\text { kann } \\
\text { ich } \\
\text { gar } \\
\text { nicht }\end{array}$ \\
\hline
\end{tabular}

\section{Widerstands- und Durchhaltefähigkeit}

Wie gut sind Sie darin bei der Stange zu bleiben oder Stress zu widerstehen, beispielsweise einen Arbeitstag durchzuhalten, sich von Widrigkeiten nicht beeindrucken zu lassen oder begonnene Aktivitäten auch dann fortzusetzen, wenn es schwer fällt?

\begin{tabular}{|c|c|c|c|c|c|c|c|}
\hline $\begin{array}{l}\text { Das ist } \\
\text { eindeu- } \\
\text { tig eine } \\
\text { Stärke } \\
\text { von mir }\end{array}$ & $\begin{array}{l}\text { Da bin } \\
\text { ich } \\
\text { besser } \\
\text { als die } \\
\text { meisten }\end{array}$ & $\begin{array}{l}\text { Das } \\
\text { kann } \\
\text { ich } \\
\text { ganz } \\
\text { gut }\end{array}$ & $\begin{array}{l}\text { Das } \\
\text { geht } \\
\text { schon } \\
\text { ir- } \\
\text { gend- } \\
\text { wie }\end{array}$ & $\begin{array}{l}\text { Das } \\
\text { klappt } \\
\text { nicht } \\
\text { im- } \\
\text { mer }\end{array}$ & $\begin{array}{l}\text { Deswegen } \\
\text { gibt es } \\
\text { schon } \\
\text { mal Pro- } \\
\text { bleme }\end{array}$ & $\begin{array}{l}\text { Da } \\
\text { müs- } \\
\text { sen } \\
\text { mir } \\
\text { andere } \\
\text { helfen }\end{array}$ & $\begin{array}{l}\text { Das } \\
\text { kann } \\
\text { ich } \\
\text { gar } \\
\text { nicht }\end{array}$ \\
\hline
\end{tabular}




\section{Selbstbehauptungsfähigkeit}

Wie gut können Sie anderen Menschen gegenüber Ihre Meinung vertreten, ohne dass Sie anderen unangemessen auf die Füße treten oder sich selbst zu klein machen? Können Sie andere manchmal dazu bringen, dass sie tun was Sie selbst möchten, andere für sich einnehmen? Können Sie sich durchsetzen, wenn Sie in einer Position sind, die das erfordert?

\begin{tabular}{llllllll}
\hline Das ist & Da bin & Das & Das & Das & Deswegen & Da & Das \\
eindeu- & ich & kann & geht & klappt & gibt es & müs- & kann \\
tig eine & besser & ich & schon & nicht & schon & sen & ich \\
Stärke & als die & ganz & ir- & im- & mal Pro- & mir & gar \\
von mir & meisten & gut & gend- & mer & bleme & andere & nicht \\
& & & wie & & & helfen & \\
\hline
\end{tabular}

\section{Gesprächs- und Kontaktfähigkeit zu Dritten}

Wie gut gelingt es Ihnen, auf andere Menschen zuzugehen, mit anderen ins Gespräch zu kommen, sich unbefangen auf Smalltalk-Gespräche einzulassen und bei anderen den Eindruck zu erwecken, dass Sie ein guter Gesprächspartner*in sind?

\begin{tabular}{|c|c|c|c|c|c|c|c|}
\hline $\begin{array}{l}\text { Das ist } \\
\text { eindeu- } \\
\text { tig eine } \\
\text { Stärke } \\
\text { von mir }\end{array}$ & $\begin{array}{l}\text { Da bin } \\
\text { ich } \\
\text { besser } \\
\text { als die } \\
\text { meisten }\end{array}$ & $\begin{array}{l}\text { Das } \\
\text { kann } \\
\text { ich } \\
\text { ganz } \\
\text { gut }\end{array}$ & $\begin{array}{l}\text { Das } \\
\text { geht } \\
\text { schon } \\
\text { ir- } \\
\text { gend- } \\
\text { wie }\end{array}$ & $\begin{array}{l}\text { Das } \\
\text { klappt } \\
\text { nicht } \\
\text { im- } \\
\text { mer }\end{array}$ & $\begin{array}{l}\text { Deswegen } \\
\text { gibt es } \\
\text { schon } \\
\text { mal Pro- } \\
\text { bleme }\end{array}$ & $\begin{array}{l}\text { Da } \\
\text { müs- } \\
\text { sen } \\
\text { mir } \\
\text { andere } \\
\text { helfen }\end{array}$ & $\begin{array}{l}\text { Das } \\
\text { kann } \\
\text { ich } \\
\text { gar } \\
\text { nicht }\end{array}$ \\
\hline
\end{tabular}

\section{Gruppenfähigkeit}

Wie gut kommen Sie in Gruppen zurecht, also in Arbeitsteams, in privaten Cliquen oder im Freundeskreis? Können Sie sich in Gruppengespräche einbringen, sich aber auch angemessen zurückhalten und den Gruppenzusammenhalt fördern?

\begin{tabular}{llllllll}
\hline Das ist & Da bin & Das & Das & Das & Deswegen & Da & Das \\
eindeu- & ich & kann & geht & klappt & gibt es & müs- & kann \\
tig eine & besser & ich & schon & nicht & schon & sen & ich \\
Stärke & als die & ganz & ir- & im- & mal Pro- & mir & gar \\
von mir & meisten & gut & $\begin{array}{l}\text { gend- } \\
\text { wie }\end{array}$ & mer & bleme & andere & nicht \\
& & & & & helfen & \\
\hline
\end{tabular}

\section{Fähigkeit zu engen Beziehungen}

Wie gut gelingt es Ihnen, enge vertrauliche Beziehungen mit nahestehenden Menschen aufzubauen und zu halten? Wie geht es Ihnen in einer Partnerschaft oder wenn Sie im engeren Familienkreis zusammen sind? Sind Sie ein Familienmensch, jemand, dem sich enge Freund*innen und Angehörige anvertrauen und auf den man sich verlassen kann?

\begin{tabular}{llllllll}
\hline Das ist & Da bin & Das & Das & Das & Deswegen & Da & Das \\
eindeu- & ich & kann & geht & klappt & gibt es & müs- & kann \\
tig eine & besser & ich & schon & nicht & schon & sen & ich \\
Stärke & als die & ganz & ir- & im- & mal Pro- & mir & gar \\
von mir & meisten & gut & $\begin{array}{l}\text { gend- } \\
\text { wie }\end{array}$ & mer & bleme & $\begin{array}{l}\text { andere } \\
\text { helfen }\end{array}$ & nicht \\
& & & & & & \\
\hline
\end{tabular}

\section{Selbstpflege und Selbstversorgung}

Wie gut sind Sie darin, auf sich und Ihr Äußeres zu achten, sich der Jahreszeit und dem Anlass entsprechen zu kleiden oder sich ausgewogen zu ernähren? Wie gut gelingt es Ihnen für Erholung zu sorgen und die gesundheitlichen Bedürfnisse Ihres Körpers wahrzunehmen? Treiben Sie regelmäßig Sport bzw. achten auf ausreichend Bewegung?

\begin{tabular}{llllllll}
\hline $\begin{array}{l}\text { Das ist } \\
\text { eindeu- }\end{array}$ & Da bin & Das & Das & Das & Deswegen & Da & Das \\
tig eine & besser & kann & geht & klappt & gibt es & müs- & kann \\
Stärke & als die & ganz & ir- & nicht & schon & sen & ich \\
von mir & meisten & gut & gend- & mer & bleme & mir & gar \\
& & & wie & & & andere & nicht \\
& & & & & \\
\hline
\end{tabular}

\section{Mobilität und Verkehrsfähigkeit}

Können Sie sich angemessen bewegen, bücken, Treppen steigen, spazieren gehen? Können Sie hingehen, wohin sie müssen, ohne Probleme einkaufen, und übliche Verkehrsmittel, wie Auto, Bus oder Bahn benutzen?

\begin{tabular}{|c|c|c|c|c|c|c|c|}
\hline $\begin{array}{l}\text { Das ist } \\
\text { eindeu- } \\
\text { tig eine } \\
\text { Stärke } \\
\text { von mir }\end{array}$ & $\begin{array}{l}\text { Da bin } \\
\text { ich } \\
\text { besser } \\
\text { als die } \\
\text { meisten }\end{array}$ & $\begin{array}{l}\text { Das } \\
\text { kann } \\
\text { ich } \\
\text { ganz } \\
\text { gut }\end{array}$ & $\begin{array}{l}\text { Das } \\
\text { geht } \\
\text { schon } \\
\text { ir- } \\
\text { gend- } \\
\text { wie }\end{array}$ & $\begin{array}{l}\text { Das } \\
\text { klappt } \\
\text { nicht } \\
\text { im- } \\
\text { mer }\end{array}$ & $\begin{array}{l}\text { Deswegen } \\
\text { gibt es } \\
\text { schon } \\
\text { mal Pro- } \\
\text { bleme }\end{array}$ & $\begin{array}{l}\mathrm{Da} \\
\text { müs- } \\
\text { sen } \\
\text { mir } \\
\text { andere } \\
\text { helfen }\end{array}$ & $\begin{array}{l}\text { Das } \\
\text { kann } \\
\text { ich } \\
\text { gar } \\
\text { nicht }\end{array}$ \\
\hline
\end{tabular}




\section{Anhang C}

\section{Fähigkeitsorientierte Arbeitsplatzbeschreibung: Mini-ICF-APP-W (Muschalla 2018a, 2018b)}

Bitte beurteilen Sie im Folgenden, in welchem Ausmaß Ihre Arbeit die beschriebenen Fähigkeitsanforderungen an Sie stellt $(0=$ trifft gar nicht zu, $1=$ trifft etwas zu, $2=$ trifft teilweise zu, 3 = trifft überwiegend zu, $4=$ trifft voll zu).

Tab. C.1 Leistungs- und Fähigkeitsanforderungen des Arbeitsplatzes (Arbeitsplatz konkret benennen)

Grad der

Anforde-

rung

1. Bei dieser Arbeit muss man sich genau an vorgegebene Vorschriften, Regeln und Routinen halten (pünktlich sein, Termine und Arbeitsabläufe einhalten)

2. Bei dieser Arbeit muss man seine Arbeit größtenteils selbst strukturieren und planen

3. Bei dieser Arbeit muss man damit umgehen können, ständig unterbrochen/gestört zu werden, oder sich an Veränderungen anpassen zu müssen

4. Bei dieser Arbeit muss man ständig viel wissen, und fachlich ständig auf dem Laufenden sein

5. Bei dieser Arbeit muss man selbst Entscheidungen treffen, die Auswirkungen haben auf Dritte oder Sachwerte (z. B. Kund*innen, Betriebsangehörige, Material, Geld)

6. Bei dieser Arbeit muss man selbst wissen was zu tun ist, und selbst die Initiative ergreifen

7. Bei dieser Arbeit muss man durchhalten können und trotz Problemen bei der Sache bleiben

8. Bei dieser Arbeit muss man seine Meinung oder eine vorgegebene Position gegenüber anderen vertreten können

9. Bei dieser Arbeit muss man sympathiewerbend und gewinnbringend mit Menschen reden können (z. B. Patienten- oder Kundengespräche)

10. Bei dieser Arbeit muss man gut mit anderen Menschen im Team zusammenarbeiten können

11. Bei dieser Arbeit muss man eine vertrauensvolle Beziehung zu einzelnen Menschen aufbauen können 12. Bei dieser Arbeit muss man sehr stark auf ein gepflegtes Äußeres und eigene Gesundheit Wert legen

13. Bei dieser Arbeit muss man viele Wege machen, sei es im Haus oder draußen

Zusatzanforderung: Bei dieser Arbeit muss man körperlich äußerst fit sein (Muskelkraft, körperliche Ausdauer, Beweglichkeit)

Zusatzanforderung: Bei dieser Arbeit muss man psychisch äußerst belastbar sein (Konzentration, Durchhaltevermögen, Flexibilität)

Zusatzanforderung: Bei dieser Arbeit hat man einen häufig wechselnden Arbeitsrhythmus (wechselnde Schichten, Tag- und Nachtschicht)

Globaleinschätzung: Mein Arbeitsplatz passt zu meinen Fähigkeiten
Funding Open Access funding enabled and organized by Projekt DEAL.

Open Access Dieser Artikel wird unter der Creative Commons Namensnennung 4.0 International Lizenz veröffentlicht, welche die Nutzung, Vervielfältigung, Bearbeitung, Verbreitung und Wiedergabe in jeglichem Medium und Format erlaubt, sofern Sie den/die ursprünglichen Autor(en) und die Quelle ordnungsgemäß nennen, einen Link zur Creative Commons Lizenz beifügen und angeben, ob Änderungen vorgenommen wurden.

Die in diesem Artikel enthaltenen Bilder und sonstiges Drittmaterial unterliegen ebenfalls der genannten Creative Commons Lizenz, sofern sich aus der Abbildungslegende nichts anderes ergibt. Sofern das betreffende Material nicht unter der genannten Creative Commons Lizenz steht und die betreffende Handlung nicht nach gesetzlichen Vorschriften erlaubt ist, ist für die oben aufgeführten Weiterverwendungen des Materials die Einwilligung des jeweiligen Rechteinhabers einzuholen.

Weitere Details zur Lizenz entnehmen Sie bitte der Lizenzinformation auf http://creativecommons.org/licenses/by/4.0/deed.de.

\section{Literatur}

AWMF (2019). Sk2 Leitlinie zur Begutachtung psychischer und psychosomatischer Erkrankungen. AWMF-Leitlinien-Register Nr. 051/029.

Balestrieri, M., Isola, M., Bonn, R., Tam, T., Vio, A., Linden, M., \& Maso, E. (2013). Validation of the Italian version of MiniICF-APP, a short instrument for rating activity and participation restrictions in psychiatric disorders. Epidemiology Psychiatric Sciences, 22, 81-91.

BMAS (2017). Arbeit weiter denken. Weißbuch Arbeit 4.0. Berlin: Bundesministerium für Arbeit und Soziales.

BMJV (2020). Gesetz über die Durchführung von Maßnahmen des Arbeitsschutzes zur Verbesserung der Sicherheit und des Gesundheitsschutzes der Beschäftigten bei der Arbeit (Arbeitsschutzgesetz - ArbSchG). Arbeitsschutzgesetz vom 7. August 1996 (BGB1. I S. 1246), das zuletzt durch Artikel 1 des Gesetzes vom 22.Dezember 2020 (BGB1. I S. 3334) geändert worden ist. Bundesministeriums der Justiz und für Verbraucherschutz.

Brütt, A.L., Schulz, H., \& Andreas, S. (2015). Replikation der psychometrischen Gütekriterien des ICF-PsychA\&P. Die Rehabilitation, 54, 38-44.

Buchholz, A., Spies, M., \& Brütt, A. L. (2015). ICF-basierte Messinstrumente zur Bedarfserfassung und Evaluation in der Behandlung von Patienten mit psychischen Störungen - ein systematischer Review. Die Rehabilitation, 54, 153-159.

Caplan, R. D., Cobbs, S., French, J. R.P., van Harrison, R., \& Pinneau, S. R. (1975). Job demands and worker health. Washington DC: National Institute of Occupational Safety and Health.

Cieza, A., Ewert, T., Üstün, T.B., Chatterji, S., Kostanjsek, N., \& Stucki, G. (2004). Development of ICF Core Sets for patients with chronic conditions. Journal of Rehabilitation Medicine, 44, 9-11.

DGPM, \& DKPM (2020). AWMF-Leitlinie zur Begutachtung psychischer und psychosomatischer Störungen. Teil II: Begutachtung der beruflichen Leistungsfähigkeit. Berlin: AWMF Arbeitsgemeinschaft der Wissenschaftlichen Medizinischen Fachgesellschaften.

DRV (2012). Leitlinie Sozialmedizinische Beurteilung bei psychischen und Verhaltensstörungen (01.08.2012). Berlin: Deutsche Rentenversicherung. https://www.deutsche-rentenversicherung.de/ Allgemein/de/Inhalt/3_Infos_fuer_Experten/01_sozialmedizin_ forschung/downloads/sozmed/begutachtung/leitlinien_rehabe duerftigkeit_psychische_stoerungen_pdf.html Stand: 03.05.2019. 
DRV (2019). Rentenversicherung in Zeitreihen. Berlin: Deutsche Rentenversicherung.

D'Zurilla, T. J., \& Goldfried, M. R. (1971). Problem solving and behavior modification. Journal of Abnormal Psychology, 78, 107-126.

Edwards, J. R., \& van Harrison, R. (1993). Job demands and worker health: three-dimensional reexamination of the relationship between person-environment fit and strain. Journal of Applied Psychology, 78, 628-648.

Endicott, J., \& Nee, J. (1997). Endicott Work Productivity Scale (EWPS): a new measure to assess treatment effects. Psychopharmacological Bulletin, 33, 13-16.

Endicott, J., Spitzer, R. L., Fleiss, J.L., \& Cohen, J. (1976). The global assessment scale. A procedure for measuring overall severity of psychiatric disturbance. Archives of General Psychiatry, 33, 766-771.

French Jr., J. R. P. (1973). Person role fit. Occupational Mental Health, $3,15-20$.

GBA. (2016). Richtlinie des gemeinsamen Bundesausschusses über die Beurteilung der Arbeitsunfähigkeit und die Maßnahmen zur stufenweisen Wiedereingliederung (Arbeitsunfähigkeits-Richtlinien) nach $\S 92$ Abs. 1 Satz 2 Nr. 7 SGB V. Gemeinsamer Bundesausschuss der Ärzte und Krankenkassen. Bundesanzeiger, BAnz AT 23. Dez. 2016 B5, in Kraft getreten am 24. Dezember 2016

Gjesdal, S., Ringdal, P.R., Haug, K., \& Maeland, J.G. (2008). Long-term sickness absence and disability pension with psychiatric diagnosis: a population-based cohort study. Nordic Journal of Psychiatry, 62, 294-301. https://doi.org/10.1080/ 08039480801984024.

Hinsch, R., \& Pfingsten, U. (2002). Gruppentraining sozialer Kompetenzen (GSK) Grundlagen, Durchführung und Anwendungsbeispiele (4. Aufl.). Weinheim: Beltz.

Jacobi, F., \& Linden, M. (2018). Macht die moderne Arbeitswelt psychisch krank - oder kommen psychisch Kranke in der modernen Arbeitswelt nicht mehr mit. Arbeitsmedizin Sozialmedizin Umweltmedizin, 53, 530-536.

Kaluza, G. (2004). Stressbewältigung. Trainingsmanual zur psychologischen Gesundheitsförderung. Berlin: Springer.

Laireiter, A.-R. (2005). Klinisch-psychologische und psychotherapeutische Diagnostik: Ähnlichkeiten und Unterschiede. In H. Bartuska, M. Buchsbaumer, G. Mehta, G. Pawlowsky \& S. Wiesnagrotzki (Hrsg.), Psychotherapeutische Diagnostik. Leitlinien für den neuen Standard. Wien: Springer.

Lawton, M.P., \& Brody, E.M. (1968). Assessment of older people: Self-maintaining and instrumental activities of daily living. The Gerontologist, 9(3), 179-186.

Linden, M., \& Muschalla, B. (2018). Type, severity and course of chronic mental disorders in general health care. Psychological Disorders and Research. https://doi.org/10.31487/j.PDR.2018.02. 004.

Linden, M., Baron, S., \& Muschalla, B. (2010). Capacity according to ICF in relation to work related attitudes and performance in psychosomatic patients. Psychopathology, 43, 262-267.

Linden, M., Baron, S., Muschalla, B., \& Ostholt-Corsten, M. (2015). Fähigkeitsbeeinträchtigungen bei psychischen Erkrankungen. Diagnostik, Therapie und sozialmedizinische Beurteilung in Anlehnung an das Mini-ICF-APP. Göttingen: Hogrefe.

Linden, M., Keller, L., Noack, N., \& Muschalla, B. (2018). Die Selbstbeurteilung von Fähigkeiten und Fähigkeitsbeeinträchtigungen im Kontext psychischer Erkrankungen: Das „Mini-ICF-APPS“. Praxis Klinische Verhaltensmedizin und Rehabilitation, 101, $14-22$.

Linden, M., Baron, S., Muschalla, B. (2009, 2015). Mini-ICF-APP. Mini-ICF-Rating für Aktivitäts- und Partizipationsstörungen bei psychischen Erkrankungen. Ein Kurzinstrument zur Fremdbeurteilung von Aktivitäts- und Partizipationsstörungen bei psychischen Erkrankungen in Anlehnung an die Internationale Klassifikation der Funktionsfähigkeit, Behinderung und Gesundheit
(ICF) der Weltgesundheitsorganisation. Bern: Huber. (Neuauflage 2015).

Löffler, G., Gerlich, C., Lukasczik, M., Wolf, H.D., \& Neuderth, S (2012). Arbeits- und berufsbezogene Orientierung in der medizinischen Rehabilitation. Praxishandbuch (3. Aufl.). Würzburg: Universität Würzburg, Arbeitsbereich Rehabilitationswissenschaften.

Meier-Credner, A., \& Muschalla, B. (2020). Kann Ungerechtigkeit bei der Arbeit krank machen? Grundannahmen, Subjektive Wahrnehmung und Person-Job-Fit. Verhaltenstherapie, 20, 256-265. https://doi.org/10.1159/000502920.

Molodynski, A., Linden, M., Juckel, G., Yeeles, K., Anderson, C., Vazquez-Montes, M., \& Burns, T. (2013). The reliability, validity, and applicability of an English language version of the MiniICF-APP. Social Psychiatry and Psychiatric Epidemiology, 48, 1347-1354.

Muschalla, B. (2014). Fähigkeitsorientierte Verhaltenstherapie bei psychischen Erkrankungen. Verhaltenstherapie, 24, 48-55.

Muschalla, B. (2018a). A concept of psychological work capacity demands-first evaluation in rehabilitation patients with and without mental disorders. Work, 59, 375-386. https://doi.org/10.3233/ WOR-182691.

Muschalla, B. (2018b). Assessing psychological work demands with an ICF-oriented concept of psychological capacities. Gruppe Interaktion Organisation, 49, 81-92. https://doi.org/10.1007/ s11612-018-0406-x.

Muschalla, B. (2019). Psychische Fähigkeiten und (Arbeits)Fähigkeitsbeeinträchtigungen beschreiben. Psychotherapie Aktuell, 04, 34-38.

Muschalla, B. (2020). Die Mini-ICF-APP-Family: Fremd- und Selbsteinschätzung von Fähigkeiten, fähigkeitsorientierte Arbeitsplatzbeschreibung und Wohnfähigkeit. Medizinischer Sachverständiger, 116, 170-182.

Newman, K. S. (2006). Chutes and ladders: navigating the low-wage labor market (S. 351). : Harvard University Press.

Nosper, M. (2008). ICF-AT-50-Psych. Entwicklung eines ICF-konformen Fragebogens für die Selbstbeurteilung von Aktivitäten und Teilhabe bei psychischen Störungen. DRV Schriften, 77, 125-128.

SGVP (2016). Qualitätsleitlinien für versicherungspsychiatrische Gutachten. Schweizerische Gesellschaft für Versicherungspsychiatrie. Qualitätsleitlinien für versicherungspsychiatrische Gutachten. http://www.iv-pro-medico.ch/fileadmin/documents/D Qualitaetsleitlinien_fuer_versicherungspsychiatrische_Gutachten 20.10.2016.pdfStand (Erstellt: 17. Okt. 2016). Zugegriffen: 6. Mai 2019

Smith, M. (2003). Sag Nein ohne Skrupel. Die neue Methode zur Steigerung von Selbstsicherheit und Selbstbehauptung. Ulm: Mvg.

Stansfeld, S. A., Clark, C., Caldwell, T., Rodgers, B., \& Power, C. (2008). Psychosocial work characteristics and anxiety and depressive disorders in midlife: the effects of prior psychological distress. Occupational and Environmental Medicine, 65, 634-642. https://doi.org/10.1136/oem.2007.036640.

Touloumakos, A. K. (2020). Expanded Yet restricted: A Mini Review of the Soft Skills Literature. Frontiers in Psychology, 11, 2207. https://doi.org/10.3389/fpsyg.2020.02207.

WHO (2001). International classification of functioning, disability and health: ICF. Geneva: World Health Organization.

WHO (2004). WHODAS II-disability assessment schedule training manual: a guide to administration. Geneva: World Health Organization.

Wiersma, D., DeJong, A., Kraaiijkamp, H. J. M., \& Ormel, J. (1990). The Groningen social disability schedule. Manual and questionnaires, 2nd version. : University of Groningen, Department of Social Psychiatry, World Health Organization.

Wittchen, H. U., Jacobi, F., Rehm, J., Gustavsson, A., Svensson, M., Jönsson, B., Olesen, J., Allgulander, A., Alonso, A., Faravelli, C., Fratiglioni, L., Jennum, P., Liebe, R., Maercker, A., van Os, J., Preisig, M., Salvador-Carulla, L., Simon, R., \& Steinhausen, 
H.C. (2011). The size and burden of mental disorders and other disorders of the brain in Europe 2010. European Neuropsychopharmacology, 21, 655-679.

Zielke, M. (2017). Die Fake-News-Connection der Versorgungsforschung bei psychischen Erkrankungen. Praxis Klinische Verhaltensmedizin und Rehabilitation, 100, 205-225.

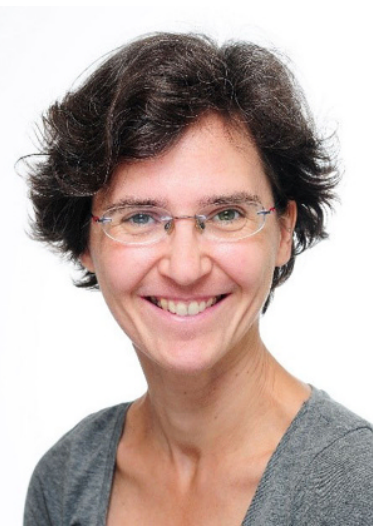

Prof. Dr. Beate Muschalla ist Verhaltenstherapeutin und Supervisorin und seit 2018 Professorin für Psychotherapie und Diagnostik an der Technischen Universität Braunschweig. Sie hat zuvor seit 2006 wissenschaftlich wie auch praktisch in der medizinischen Rehabilitation gearbeitet, war Leitende Psychologin der Deutschen Rentenversicherung Bund und hat mehrere klinische Studien geleitet, in Kooperation mit dem Bundeswehrkrankenhaus, Rehabilitationskliniken und Hausärzten. Sie forscht zu Arbeitsängsten, Arbeitsfähigkeit, Verbitterung und Weisheit sowie Betrieblicher Gesundheitsförderung und Wiedereingliederung (BEM). 